\title{
Intermédialités
}

Histoire et théorie des arts, des lettres et des techniques

Intermediality

History and Theory of the Arts, Literature and Technologies

\section{Survivances du Voyage en Italie}

\section{André Habib}

Numéro 5, printemps 2005

Transmettre

Transmitting

URI : https://id.erudit.org/iderudit/1005492ar

DOI : https://doi.org/10.7202/1005492ar

Aller au sommaire du numéro

\section{Éditeur(s)}

Centre de recherche sur l'intermédialite

ISSN

1705-8546 (imprimé)

1920-3136 (numérique)

Découvrir la revue

Citer cet article

Habib, A. (2005). Survivances du Voyage en Italie. Intermédialités /

Intermediality, (5), 61-80. https://doi.org/10.7202/1005492ar

\section{Résumé de l'article}

Cet article se penche sur Viaggio in Italia (1954) de Roberto Rossellini. L'auteur montre que l'enjeu principal de ce film consiste à démonter et remonter, par un jeu subtil de réfractions et de mise en abîme, l'idée et la pratique de l'héritage, de la transmission d'une tradition (en passant par la tradition des voyages en Italie), et ce, à partir d'une rhétorique de l'écart et du faux-raccord, de la survivance, en creux, du passé dans les ruines du temps. Le sens de ce qui reste, alors, devient le sens d'une perte de sens du monde qui révèle dans sa complexité ce qui a été perdu. Si l'on peut parler de transmission et d'héritage ici, ce n'est qu'en relisant cette perte de sens comme ce dont on hérite et qui serait à redécouvrir continûment. C'est en ceci que Rossellini, avec ce film, pose les premières pierres d'un cinéma moderne qui tentera, depuis, d'en transmettre l'héritage. 


\title{
Survivances du Voyage en Italie
}

\author{
ANDRÉ HabiB
}

E

n avril 1955, Jacques Rivette, alors jeune critique aux Cahiers du cinéma, d'une plume vive et affectueusement prophétique, s'écriait à propos du film de Roberto Rossellini Viaggio in Italia (1954) : «Ce film ouvre une brèche et le cinéma tout entier devra y passer sous peine de mort. [...] Voilà notre cinéma, à nous qui nous apprêtons à notre tour à faire des films (vous l'ai-je dit, c'est pour bientôt peut-être) ${ }^{1}$. » Cette «Lettre sur Rossellini » paraît aujourd'hui précieuse parce qu'elle marque la mesure d'une volonté de filiation que les films de la Nouvelle Vague, ceux de Rohmer, de Godard, de Rivette lui-même, n’ont pas trahie par la suite ${ }^{2}$. Ces critiques bientôt cinéastes des Cahiers jaunes se sont pressentis, dans l'immédiateté de cette reconnaissance, dans l'évidence du film, comme les héritiers d'une œuvre dont ils porteraient, au cœur de leurs œuvres respectives, avec des gestes de réappropriation très divers, la trace: Voyage en Italie leur présentait, pourrait-on dire, le passé de leur avenir.

Ce qui frappe en relisant cette Lettre, c'est moins l'opportunité stratégique d'une attribution de nouveauté - le film, à l'époque, fut reçu avec indifférence - , que l'ambiguïté de cet héritage qui ne semble, pour reprendre en la détournant l'expression de René Char, «précédé d'aucun testament ${ }^{3}$ ». Car il s'agit bien de voir - et cet article tentera de le rendre visible - que dans ce

1. Jacques Rivette, «Lettre sur Rossellini», Cahiers du cinéma, n 46, avril 1955, repris dans Antoine de Baecque (éd.), La politique des auteurs, IV. Petite anthologie des Cahiers du cinéma, Paris, Éditions Cahiers du cinéma, 2001, p. 75.

2. Alain Bergala, dans son ouvrage sur Voyage en Italie, insiste beaucoup sur la liaison entre ce film et les films de la Nouvelle Vague. Voir Alain Bergala, Voyage en Italie, de Roberto Rossellini, Paris, Éditions Yellow Now, 1990, en particulier p. 25-26.

3. René Char, cité dans Hanna Arendt, Between Past and Future: Eight Exercises in Political Thought, New York, The Viking Press, 1968, p. 3. 
film, l'enjeu principal consiste à démonter et remonter, par un jeu subtil de réfractions et de mise en abîme, l'idée et la pratique de l'héritage, de la filiation, de la transmission d'une tradition, et ce, à partir d'une rhétorique de l'écart et du faux-raccord, de la survivance, en creux, du passé dans les ruines du temps. Ce dont ils hériteraient serait, précisément, une mise en crise de l’idée de transmission. En effet, si «ce film ouvre une brèche», c'est en étant précisément lui-même le signe manifeste d'une brèche entre le passé et l'avenir, entre l'homme et le monde. C'est cette brèche que tout le cinéma moderne n'a cessé par la suite - d'Antonioni à Pasolini, des nouvelles vagues européennes jusqu'à Akerman, Angelopoulos et Tarr - d'approfondir et de décliner jusqu'à l'épuisement et au vertige toutes les modalités.

Rossellini pose peut-être dans ce film les premières pierres, devenues pierres de touches, du cinéma moderne: réinvention de l'esthétique du fragment et de l'ellipse, art du déphasage entre gestes et paroles, fractionnement du sujet redevenu étranger au monde, mise en ruine de l'enchaînement propre au récit narratif classique, etc. ${ }^{4}$ Il est sans doute significatif pour le propos que je défendrai ici que Rossellini ait auparavant, et au plus près, filmé les ruines de la guerre, à Rome, Florence, à Berlin, et qu'il ait immédiatement reconnu (ce sera le legs du néo-réalisme), le nouveau régime d’images que la guerre introduisait de force dans le cinéma, et qui lui donna une valeur, auparavant méconnue, d'actualité pour l'histoire5. Du passé-présent traumatique de la guerre qui figure dans Roma aperta (1946), Paisà (1947) et Germania anno zero (1948), au trèspassé antique de Pompéi et de Naples dans Viaggio in Italia, il y a sans doute plus qu'un pas: le franchir, ne fût-ce que de façon heuristique, permet néan-

4. C’est l'idée que développe également, mais différemment, Alain Bergala, «Roberto Rossellini et l'invention du cinéma moderne », dans Roberto Rossellini, Le cinéma révélé, Paris, Éditions de l'Étoile, coll. «Champs Contre-champs. Flammarion», 1984, p. 7-30.

5. C'est Gilles Deleuze, à la suite d'André Bazin, qui insiste sur ce «nouveau régime de signes» que le néo-réalisme instaure dans la cinéma: Voir Gilles Deleuze, Cinéma 1. L'image-mouvement, Paris, Éditions de Minuit, coll. «Critique», 1983, p. 284290, et Cinéma 2. L’image-temps, Paris, Éditions de Minuit, coll. «Critique», 1985, p. 737. Quant au caractère inaugural du film de Rossellini pour l'histoire du cinéma moderne, il ne fait pas toujours école (certains auteurs lui préféreront Citizen Kane (1941) d'Orson Welles ou Hiroshima mon amour (1959) d'Alain Resnais). Il ne s'agit pas de remettre des palmes, mais de voir comment une position à la fois critique et cinéphilique s'est jouée dans le sillon de ce film et de constater que cette œuvre marque un moment déterminant pour la suite du cinéma moderne quant à l'idée de transmission. 
moins de rajouter une couche de sens à la stratification des temporalités dont ce film est porteur et, au delà, de relire à «rebrousse-poil» la modernité de ce film à partir de la fracture dans l'imaginaire que la guerre avait engendrée.

Partir de cette fracture, traiter des restes, des éclats qu'elle a laissés dans son sillon, c'est rendre compte d'une disparition. Mais c'est aussi remarquer les survivances, les fragments d'une forme, d'un échange, d'une pratique ou d'une question, à l'intérieur d'un dispositif historique, ou d'une configuration de savoir qui la dépasse, la déborde, l'anachronise ${ }^{6}$. Pour le dire plus simplement, plutôt que de liquider tout bonnement l'héritage, Rossellini met en scène sa mise en question, à partir d'un déphasage entre ses personnages et ce dont ils sont censés hériter. C'est le sens d'une perte de sens de la transmission et de l'héritage qui se fait jour alors, comme un lot qui échoit, qui est transmis aux rescapés.

Je tenterai dans les pages qui suivent de réfléchir non pas tant sur la singulière postérité de ce film (qui eut beaucoup d'enfants), mais de localiser le sens délocalisé et paradoxal de l'héritage, de la transmission et de la tradition (en passant par la tradition littéraire des voyages en Italie), qui s'y trouve mis en jeu, entre rupture et survivance, entre le «temps pur» des ruines et la ruine du temps. J'interrogerai enfin le sens de cette perte de sens, inscrite dans le matériau même du film, en tentant de voir l'occasion de sens qu'elle représente pour penser une «autre histoire».

6. Rossellini a toujours été sensible à ces décalages. Déjà, dans Germania anno zero, le discours d'Hitler entendu dans les décombres de la chancellerie marquait l'anachronie des ruines, comme, ailleurs dans le film, les échos survivant du discours eugéniste. 
«The testament, telling the heir what will be rightfully his, wills past possessions for a future.»

(Hanna Arendt, Between Past and Future ${ }^{77}$ )

"We should be able to get rid of this inheritance business in no time.»

(Alex Joyce dans Viaggio in Italia)

D'où advient-il qu'un reste nous advienne et fasse événement, dès lors que les voies de médiation et les instances institutionnelles qui régulaient les échanges entre le passé et l'avenir ont cessé d'être prégnantes? Comment accueillir un héritage, à partir du moment où nous nous situons dans ce qu'Hanna Arendt a appelé «the gap between past and future» («la brèche entre le passé et le futur $\left.{ }^{8} »\right)$ et que l'héritage a cessé de résonner comme une pratique de sens et le lieu d'une reconnaissance symbolique pour se situer dans le monde? Comment hérite-t-on des traces qui signent, par la force ambiguë de leur présence, une disparition, une fracture entre soi et le monde? S'agit-il encore d'un héritage? De quel ordre?

Toujours, ce dont on est l'héritier appartient à quelqu'un ou quelque chose qui n'est plus: il serait absurde, à la lettre, de devenir l'héritier d'un vivant! Mais l'héritage est aussi une façon de maintenir vivant ce qui n'est plus, et ce, pour le futur: le souvenir des morts, les biens des trépassés. C'est ainsi que, pour Arendt, le testament «assigne une place au vivant», garantissant une continuité historique et la conscience de soi comme sujet historique. Mais si nous cessons de nous représenter le sens, le temps et l'histoire comme ce qui nous échoit par destination, dans le flux continu des jeux de générations, d'écoles, de filiations et de traditions, alors comment nous représenter le sens de ce qui reste? Ces traces peuvent-elles encore être traitées, si le fil de la tradition est reconnu comme rompu? Rossellini, avec Viaggio in Italia, se place au centre de ce questionnement et ce, sans doute, à l'occasion d'un hasard fâcheux, dont il aura saisi toute l'occasion, et dont il est sans doute bon de rappeler les circonstances (d'autres circonstances auraient peut-être produit un autre film).

En 1953, Rossellini et toute une équipe de tournage avec, à son bord, deux vedettes hollywoodiennes de renom, Ingrid Bergman (l'épouse du cinéaste) et

7. Hanna Arendt, Between Past and Future, p. 5.

8. Il s'agit du titre de la préface de Between Past and Future. 
George Sanders, se retrouvent à Naples pour y réaliser un film d'après le roman Duo (1930) de Colette. Sur le point de tourner, on apprend que les droits du récit n’ont pu être débloqués. Ce père du néo-réalisme - qui n’aura eu de cesse, depuis la fin des années 1940, avec Stromboli, de tenter de se dégager de cette formule contraignante - s'employa alors à «improviser» le plus rigoureusement du monde ce film, à partir d'un synopsis de quelques pages et en suivant une intuition devenue proverbiale, qui fera, à l'image de son tournage, la part belle aux errances, au vide, à l'indécision des parcours, au temps qu'on laisse remonter.

Katherine et Alex Joyce ont hérité d'une propriété à Naples d'un certain oncle Homère, qui y avait élu domicile durant la seconde grande guerre. Dès le départ, ce couple de Londoniens que l'on voit rouler en Bentley présente ce voyage comme une excursion dont ils se seraient passés (le mari en particulier): passage protocolaire pour se débarrasser d'un héritage dont ils ne sauraient que faire ( We should be able to get rid of this inheritance business in no time»), et dont l'occasion de vacances pèse plus qu'elle ne paraît reposer («This is the first time we've been really alone ever since we married... - Yes, I suppose it is...", répond le mari, grincheux).

Arrachés à leurs positions sociales qui les maintenaient dans des espaces sécurisants, Katherine et Alex se retrouvent étrangers l'un à l'autre dans une terre également étrangère (d'où le titre en anglais, Strangers, qui se décline de multiples façons). On pourrait dire que, n'ayant pas d'enfants et avec des liens d'amour passablement émoussés, leur seuls rapports étaient de convenance sociale («At home, everything seemed so perfect»), et l'ironie du mari n'y changera rien: "Now that we're strangers, we can start all over at the beginning... rather amusing, don't you think? " Ce nouveau départ qui s'annonce avec cette pointe cynique sera, en fait, l'occasion d'une série de découvertes ( I I didn't know you were so fond of women », "I didn't know you were so keen on museums », etc.) et la montée graduelle du mépris ${ }^{9}$. Katherine et Alex étant libres de se voir en face comme des étrangers, le couple tombera en miettes (c'est également la condition de leur salut respectif).

On pourrait dire que la méprise - il y en a de multiples dans ce film porterait déjà sur le sens du voyage. Pour Alex, il s'agit d'un «business trip», dont le seul enjeu consiste à faire une vente rapide et fructueuse; Katherine,

9. Nous aurons l'occasion de souligner ailleurs dans ce travail les analogies entre le film de Rossellini et Le mépris (1963) de Jean-Luc Godard. 
pour sa part, voudrait qu'il s'agisse d'un "pleasure trip», d'où son excitation à l'idée de visiter Pompéi et de découvrir Naples, à voir son mari prendre du repos, etc. Cette mésentente produira deux feuilles de routes séparées: elle tracera la sienne dans le musée national de Naples, en haut du Vésuve, dans la grotte des Sibylles; lui, du côté de Capri, des soirées mondaines, à la recherche de plaisirs qu'il ne trouvera pas. Au final, on ne saura pas si un acheteur s'est prononcé sur la propriété, les visites de Katherine la bouleverseront plus qu'elles ne lui procureront du plaisir. C'est autre chose, d'inattendu, qui les attend.

Vers la fin du film, au moment où ils constateront que leur divorce est inévitable, ils seront contraints par un ami archéologue de visiter le site de Pompéi où ils assisteront à la «résurgence » d'un couple, enterré sous la lave, dont on vient de couler un moule. Katherine, devant ce spectacle, fondra en larmes et quittera éplorée le chantier des fouilles. Le couple errera alors dans les ruines de Pompéi, et une fois lancé dans les rues de Naples, leur voiture sera à nouveau forcée de s'immobiliser, arrêtée par une foule mobilisée pour une fête religieuse (la fête de Saint-Janvier) ${ }^{10}$. Emporté dans la mêlée, par l'afflux populaire, et par le miracle qui a lieu (un homme retrouve la vue, un autre l'usage de ses jambes), ils se trouvent frappés par une grâce inattendue et retombent dans les bras l'un de l'autre. Ils font leur auto-critique et se confessent leur amour, révélé, comme par miracle (fig. 1).

On aura reconnu ici le parcours (vers la grâce) du personnage-type rossellinien (il suffit de penser à la fin de Stromboli ou de Germania anno zero) qu'il a lui même décrit en ces termes: "un être tout petit au-dessous de quelque chose qui le domine et qui, d'un seul coup, le frappera effroyablement au moment précis où il se trouve librement dans le monde, sans s'attendre à quoi que ce soit ${ }^{11}$ ». On pourrait dire que, comme un bâtiment en ruines qui aurait perdu toute valeur d'échange et d'usage, une fois dépouillé de sa laque et de sa couverture sociale, le couple se révèle à lui-même comme ruine, exposé à la sédimentation du temps ${ }^{12}$. Les ruines de Pompéi et le couple désenseveli

10. Dès le début et tout au long du film, les parcours en voiture sont ponctués d'arrêts, d'attente, à l'image du film qui est, lui aussi, ponctué de longues plages de latences, de suspension. La voiture représente aussi un filtre derrière lequel le couple se protège : une fois sortis et mêlés à la foule, ils se retrouvent «librement dans le monde », selon le mot de Rossellini.

11. Cité dans Alain Bergala, Voyage en Italie, p. 47.

12. Jean-Louis Déotte explique bien cette relation entre valeur d'usage et valeur d'exposition, propre à la fois à l'art et aux ruines. On pourrait dire (et plusieurs l'ont dit), 


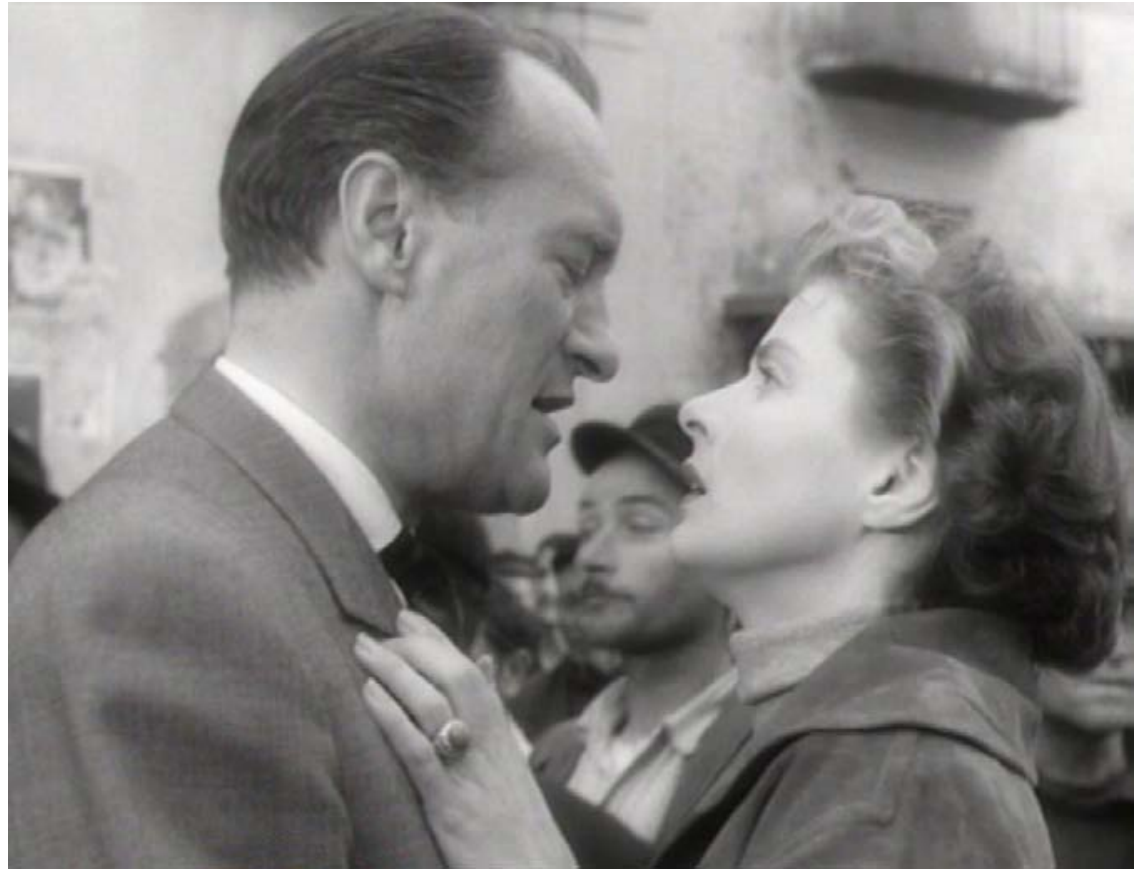

Fig. 1. Roberto Rossellini, Viaggio in Italia, 1954 (avec l'aimable autorisation de Renzo Rossellini).

renvoient au couple sa propre image, en abîme. Or, ce dénuement est aussi la condition du dénouement: c'est en suivant cette voie du dépouillement, le long de ce temps horizontal stratifié, que les deux seront soudain frappés, « effroyablement», par cette chose qui «les domine» (verticalement), comme un instantané épiphanique.

Afin de bien mesurer la portée de cet «instant miraculeux », il nous faut repartir de cette question de l'héritage, qui sert de prétexte narratif au film, afin d'envisager sa relation avec le couple qui se délite. Les Joyce héritent en effet d'une propriété habitée sur tous les plans: habitée par une famille de serviteurs dont le mari, ami de l'oncle, travaille aux excavations de Pompéi, habitée par les souvenirs de l'oncle que souligne le guide leur faisant faire le tour de la

que la ruine est, partant de là, l'œuvre d'art par excellence ou, plus exactement, que toute œuvre est toujours déjà ruine, en tant que «mode d'apparaître» et d'exposition. Voir Jean-Louis Déotte, Oubliez! Les ruines, l'Europe, le musée, Paris, Éditions de L'Harmattan, coll. «La philosophie en commun», 1994, p. 33-49. 
propriété (les toiles, les meubles, la patience collectionneuse), habitée, enfin, par sa position géographique: sur la côte napolitaine, la terrasse donne sur Capri, Pompéi, le Vésuve, etc. (fig. 2) Tous ces lieux sont littéralement enceints d'une lourde tradition, historique, littéraire, artistique. Cette propriété, en d'autres mots, est tout sauf un non-lieu, un espace de transit. Sa valeur d'échange est en quelque sorte, de partout, écrasée par sa valeur objective d'histoire et sa valeur subjective d'ancienneté, d'où l'incompréhension de l'ami devant la volonté du couple de s'en débarrasser.

C'est qu'il y a, bien entendu, deux façons d'entendre l'héritage, deux valeurs au moins qu'il peut prendre. En héritant d'un titre de propriété, les Joyce héritent en effet d'une entité légale et commerciale. C'est une permutation financière, un transfert de biens matériels, potentiellement liquidables. Considéré du point de vue d'un échange commercial, strictly business, l'héritage est une bonne affaire, qui n'engage l'héritier qu'en autant qu'il est le destinataire et le vendeur d'un titre. Mais on pourrait considérer un autre sens de l'héritage, dans le sens d'un don, venu du passé, pour l'avenir. L'héritage n'est alors pas quelque chose qui s'échange, se liquide, mais qu'on porte et qu'on transmet: il est, à proprement parler, une destination, à la fois comme réclamation venue du passé et comme promesse d'avenir. L'héritier, compris dans ce sens, n’est pas un passeur passif, mais un médiateur actif, interprète du passé (des morts), pour des vivants qui viendront après lui. Héritage et transmission sont, en ce sens, coextensifs l'un à l'autre, dans la mesure où le sujet doit réitérer certains traits propres à une tradition qu'il réactualise, dans la mesure où il y participe. Recevoir un héritage et se reconnaître dans une tradition, participent d'un même paradigme de la transmission, où le présent réfléchit le passé pour l'avenir.

C'est dans l'écart entre ces deux acceptions de l'héritage que se situe le nœud du film de Rossellini, ou plutôt, c'est en se méprenant sur ce dont ils héritent que l'étrangeté des Joyce à eux-mêmes, au lieu et au temps, se dénouera, comme choc, comme fulgurance. Le moment épiphanique du couple, celui de Katherine en particulier, survient lorsqu'ils auront subi jusqu'au bout, jusqu'à l'intolérable, leur non-appartenance au temps compris comme présent toujours débordé par le passé et l'avenir. Cette réalisation, lente, se jouant dans les moments de latence où le temps de l'action est suspendu, survient à force de se heurter à la ville et à ses habitants, au rythme et à la logique de son temps. Si Rossellini disait de Naples qu'elle «présentait le sentiment de la vie éternelle», c'est précisément parce que, partout les vivants jouxtent les morts, partout, vie et mort se relaient. 


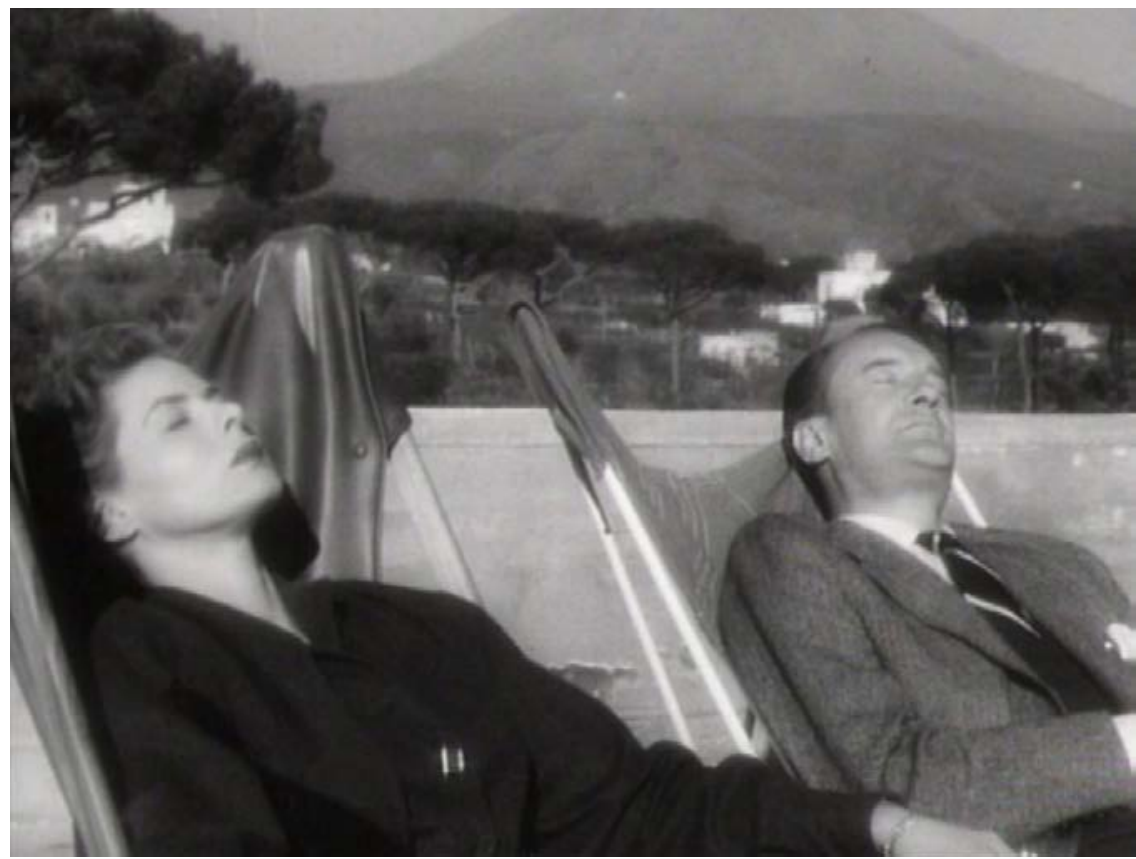

Fig. 2. Roberto Rossellini, Viaggio in Italia, 1954 (avec l'aimable autorisation de Renzo Rossellini).

Durant l'une de ses multiples pérégrinations dans les rues de Naples en voiture, Katherine voit l'un à la suite de l'autre un berceau et un corbillard, conjuguant dans un même champ du regard, les deux extrémités du destin humain. Son regard insiste sur le nombre considérable de femmes dans les rues qui sont enceintes (autre façon de penser l'engendrement et la filiation). Dans les catacombes, Natalia montre à Katherine le squelette d'un individu, mort il y a 300 ans, dont elle s'occupe et à travers lequel elle prie, pour son propre frère qui mourut en Grèce durant la guerre et dont on n'a jamais pu retrouver le corps. Dans le musée de Naples, le guide dit à Katherine que la statue de la fille de Zeus ressemble comme deux gouttes d'eau à sa fille et dans la grotte des Sibylles, un autre guide indiquera du doigt une des stations du voyage d'Ulysse ainsi que le lieu où les troupes anglaises débarquèrent durant la seconde guerre mondiale, faisant se jouxter sur un même plan, dans une même phrase, le présent, le passé, et un temps mythologique. De plus, on pourrait dire que la fête religieuse à laquelle assiste le couple est une forme d'inscription (inscrite dans le calendrier) d'un retour nécessaire et inéluctable, en tant que moment de réaffirmation de la foi (passé et à venir). La fête de Saint-Janvier a ceci 
également de singulier, qu'elle programme la tenue réglée d'un miracle et combine donc un événement parfaitement exceptionnel à la répétition du temps calendaire. En somme, on entretient avec le passé des rapports de vie, la communauté est maintenue par une série de liens imaginaires et symboliques, des récits de filiation, de scénarios d'engendrement ${ }^{13}$, etc. De la même façon, c'est avec une délectation que ne peuvent partager les Joyce que leur ami les invite à assister à l'exhumation du couple de Pompéi, comme s'il s'agissait d'assister à une naissance.

On pourrait dire que tous les habitants de Naples, des serviteurs aux vestiges aristocratiques qu'ils rencontrent lors d'une soirée mondaine, se raccrochent à des figures qui leur assurent une suture avec le temps. En ce sens, même l'ironie rassurée de la duchesse qui prétend que: "We here are all ship-wrecked», dit quand même l'appartenance au navire qui, bien que chaviré, demeure encore un repère de navigation imaginaire dans le temps.

Or, c'est précisément cette suture dans le temps qui est montrée comme liquidée ou rompue tout au long du film, à la fois chez les deux principaux protagonistes et, à travers eux, sur un plan plus formel, dans l'économie du regard et des cadrages. Après avoir perdu leurs liens de société (rattachés à un lieu, Londres) qui leur assuraient une continuité (imaginaire, symbolique) dans le monde, leur délocalisation (ou leur déterritorialisation) les plonge dans une inquiétude du temps. Alors que de partout le temps fait lien avec l'autre, il fait faux-raccord avec eux.

Pour reprendre la formule que Jean-Luc Godard avait employée à propos du mépris (1963) - adaptation du roman de Moravia, mais reprise avouée du film de Rossellini — , ces personnages sont des «naufragés du monde occidental, des rescapés du naufrage de la modernité ${ }^{14} »$. Ces naufragés, dira-t-on, ont

13. Walter Benjamin et Asja Lacis constataient que, à Naples, la «porosité» des habitations faisait en sorte que le domaine privé est constamment traversé par la vie de la communauté (et vice versa), que l'intime et le collectif s'interpénètrent de façon essentielle. Voir Walter Benjamin, Asja Lacis, «Naples» [1925], dans Walter Benjamin, Reflections: Essays, Aphorisms, Autobiographical Writings, Peter Demetz (éd.), trad. Edmund Jephcott, New York, Schoken Books, 1986 [1978], p. 163-173.

14. Jean-Luc Godard, «Le mépris», Cahiers du cinéma, $\mathrm{n}^{\circ}{ }_{146}$, août 1963, repris dans Godard par Godard, tome 1, Alain Bergala (éd.), Paris, Éditions Cahiers du cinéma, 1998 [1985], p. 249. Godard semble, dans Le mépris, avoir repris la situation dramatique de Viaggio in Italia (un couple dans le sud de l'Italie, entre Naples et Capri, s'effrite), tout en accentuant l'intertexte homérien qui y circulait. Une affiche du film (trois en fait) 


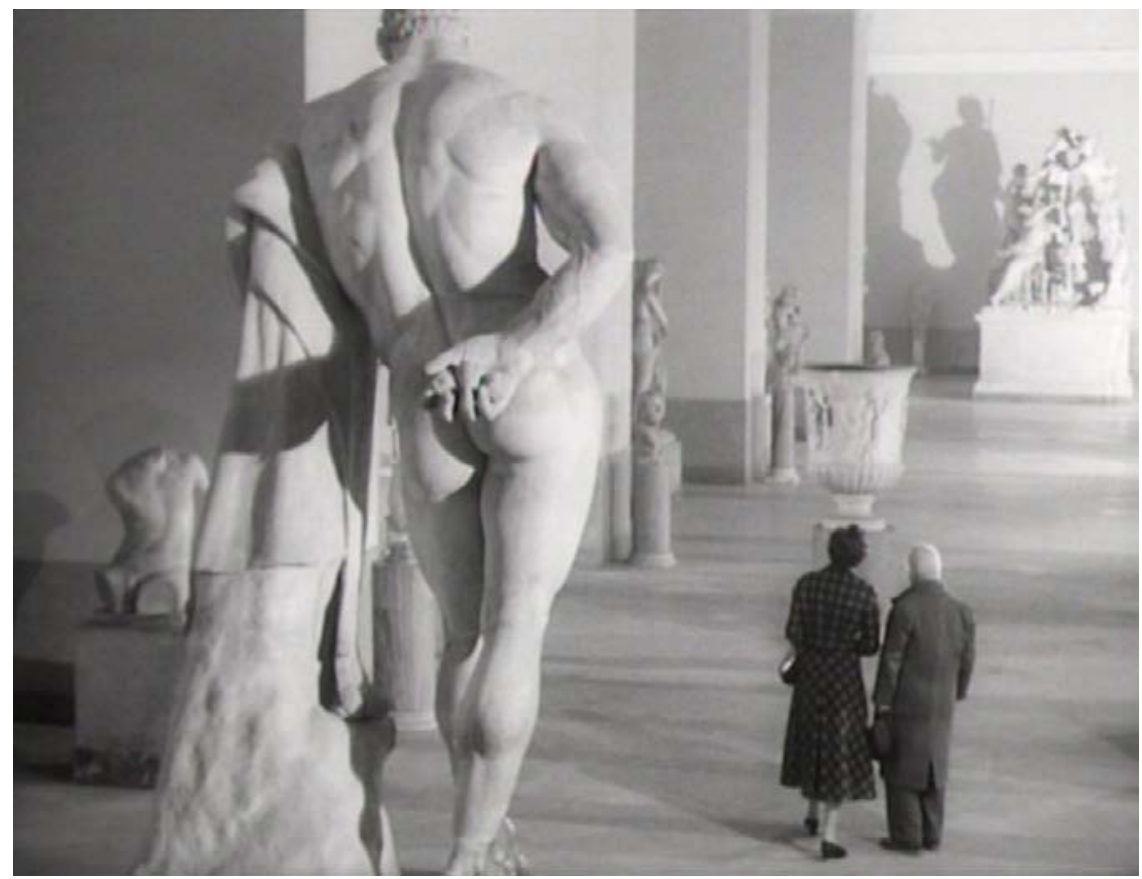

Fig. 3. Roberto Rossellini, Viaggio in Italia, 1954 (avec l'aimable autorisation de Renzo Rossellini).

perdu jusqu'au sens du naufrage, comme naufrage ${ }^{15}$. On dira que c'est l'apprentissage de ce naufrage en tant que naufrage dont le film trace le chemin.

La réussite de Rossellini, la plus déterminante pour la suite de l'histoire, est d'être parvenu à faire passer cette brèche dans le temps, à l'intérieur même de l'enchaînement - devenu désenchaîné - de son film. La brèche est passée

y apparaît à un moment, après la scène de l'audition. Les personnages sortent de la salle et, du trottoir, on voit plusieurs affiches publicitaires du film, qui est également annoncé sur la marquise du cinéma. Godard rend au film un double hommage puisqu'il renvoie réflexivement, dans la construction de son plan, à l'intelligence du cadrage rossellinien: c'est «dans le dos» des personnages que les affiches de Viaggio nous sont montrées.

15. Martin Heidegger dira un peu dans le même sens: «Mais encore pis s'annonce dans le défaut de dieu. Non seulement le dieu et les dieux se sont enfuis, mais la splendeur de la divinité s'est éteinte dans l'histoire du monde. Le temps de la nuit du monde est le temps de la détresse, parce qu'il devient de plus en plus étroit. Il est même devenu si étroit, qu'il n'est même plus capable de retenir le défaut de dieu comme défaut.» (Martin Heidegger, «Pourquoi des poètes", dans Chemins qui ne mènent nulle part, trad. Wolfgang Brokmeir, Paris, Éditions Gallimard, coll. «Tel », 1962 [1949], p. 324) 


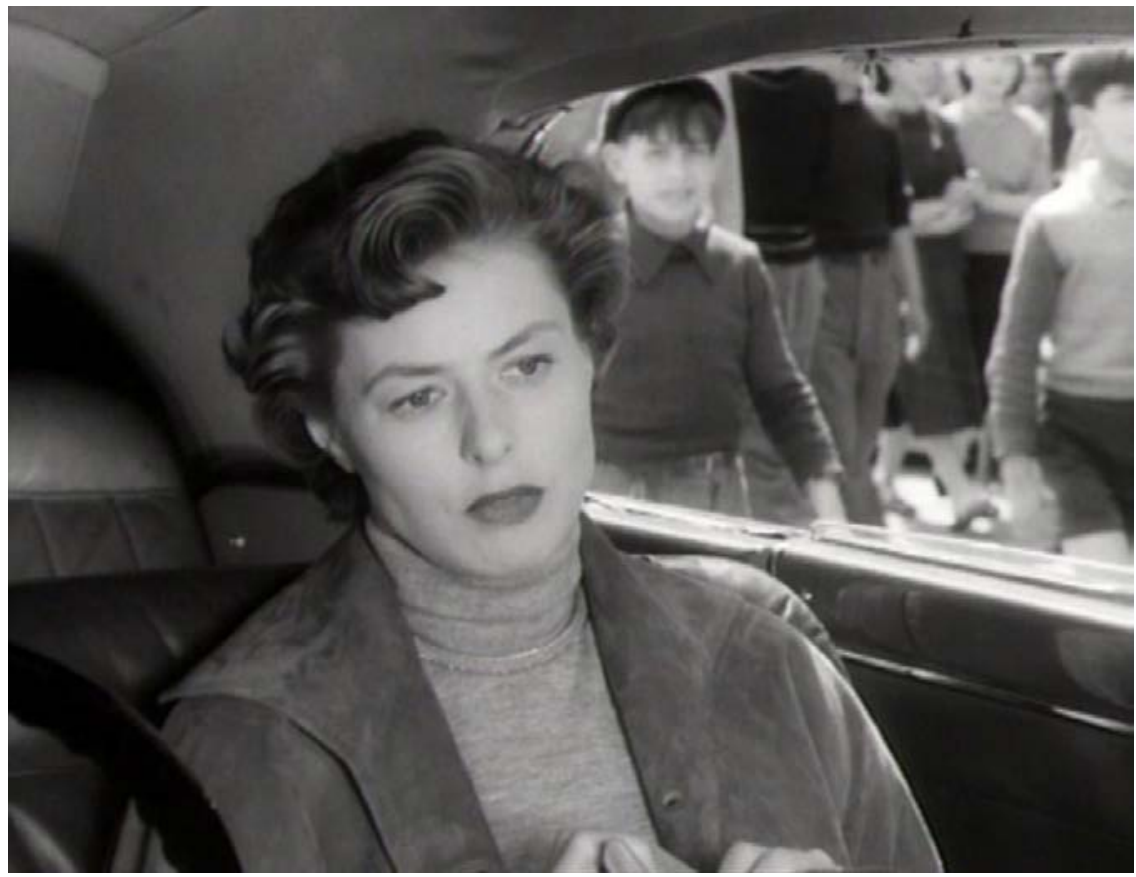

Fig. 4. Roberto Rossellini, Viaggio in Italia, 1954 (avec l'aimable autorisation de Renzo Rossellini).

dans les raccords, dans les cadrages et ce, par l'élaboration de deux plans distincts (parfois à l'intérieur du même cadre, parfois à l'aide d'une coupe). Nombreuses sont les scènes dans ce film où la caméra décide de décadrer les personnages et de faire figurer, dans leur dos, les signes de la filiation ou du passé auquel ils demeurent aveugles. Ou encore, peu avant que Katherine ne sorte du musée de Naples, un plan de grue opère un mouvement vertical, cadrant l'immense Hercule Farnese de telle sorte que la sculpture semble la surplomber du regard (c'est alors, aussi, le film qui la surplombe comme l'autre du regard) (fig. 3). Ou encore, vers la fin du film, dans la voiture, pendant que Katherine parle à Alex des enfants qu'ils n'ont pas eus, fait auquel elle attribue l'échec de leur mariage, un enfant passe dans le cadre de la fenêtre, dans son dos (nous, les spectateurs, bien entendu, le voyons) (fig. 4).

De façon encore plus significative on pourrait remarquer que, tout au long du film, tant dans le musée que dans les catacombes que devant le corps de plâtre désenseveli à Pompéi, Rossellini opère une séparation radicale entre les 

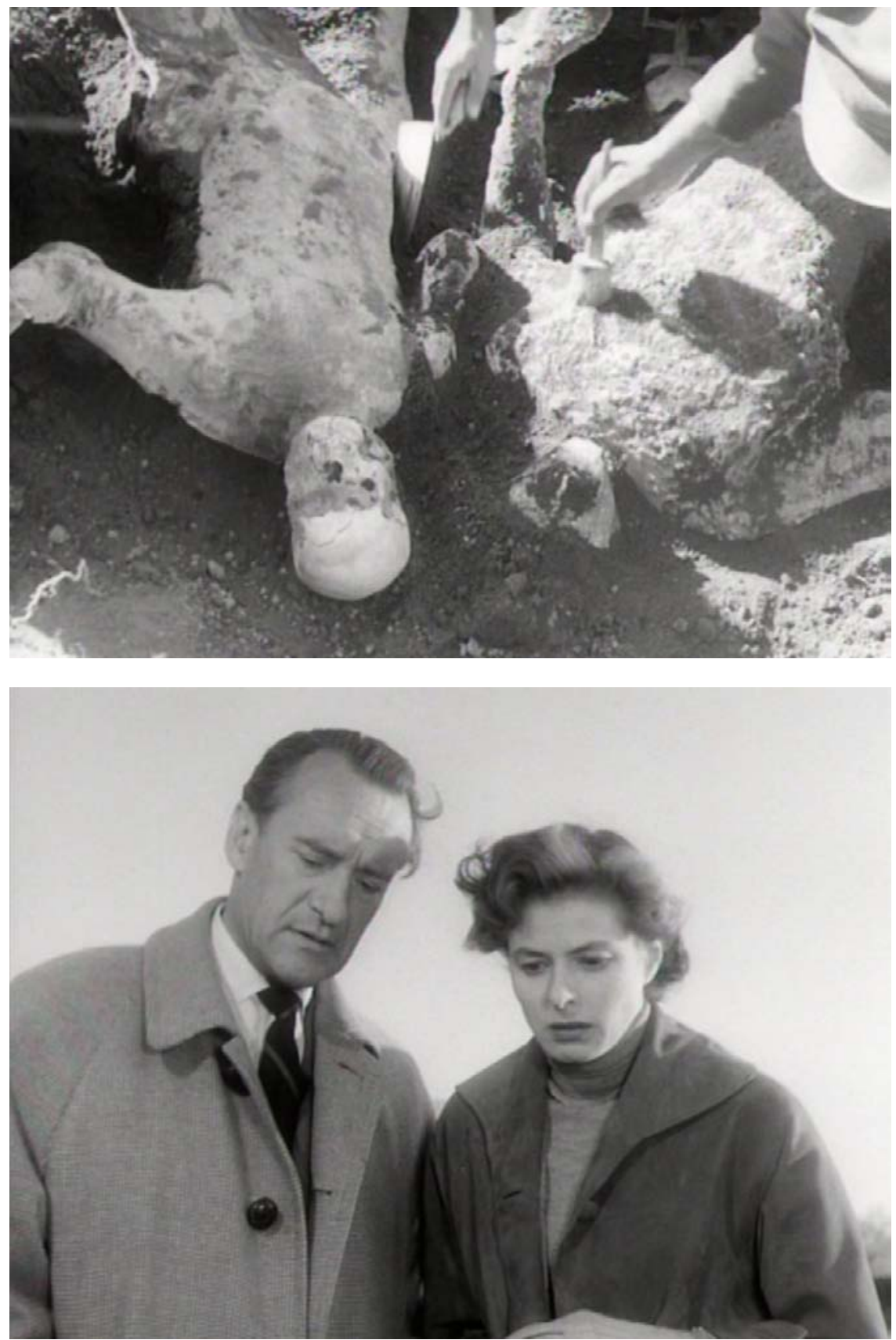

Fig. 5-6. Roberto Rossellini, Viaggio in Italia, 1954 (avec l'aimable autorisation de Renzo Rossellini). 
personnages et les objets énigmatiques du passé (comme autrefois dans les tableaux de la Renaissance on plaçait les objets sacrés et les objets profanes sur deux plans distincts ${ }^{16}$ ). Son découpage fait volontairement violence à la logique du regard, en rompant l'axe naturel entre le sujet percevant et l'objet perçu (fig. 5-6). En éloignant les œuvres ou les traces du passé du regard des personnages, Rossellini invente et cristallise ce que Alain Bergala a appelé le «fauxraccord comme non-raccord ontologique ${ }^{17}{ }^{\prime}$. Ce qui apparaît alors c'est une perte de sens liée à la faillite d'une suture imaginaire entre le soi, l'autre et le passé.

Cette perte de sens correspond à une impossibilité de se situer dans la logique d'un temps et d'une mémoire qui garantirait la continuité entre le passé et l'avenir. Ne retrouve-t-on pas, là, la formule de Tocqueville : «Le passé n’éclairant plus l'avenir, l'esprit marche dans les ténèbres ${ }^{18}$ »? Or, c'est bien dans l'immédiat de cette rencontre disjointe avec le temps (les multiples passés), quand s'éloignent tous les guides-médiateurs, que le Voyage en Italie prend tout son sens et sa valeur, justement parce qu'il offre, à partir des restes du passé et de leur trace, la perte de sens en tant que reste et comme sens qui échoit à la conscience, comme un défi à devoir se mouvoir dans la brèche, brèche que ces restes et traces font resurgir par leur seule présence (fig. 7).

Ne serait-il pas possible de dire que Rossellini se pose ici la question que s'est posée, différemment, toute la littérature moderne, en passant par Joyce, Faulkner, Sarraute, Robbe-Grillet, à savoir : comment traiter dans la langue l'écart entre l'écriture, l'expression et le monde, dès lors que le rapport à la tradition ne s'envisage plus comme allant de soi, et que, selon la célèbre formule de Beckett, «tout langage est un écart de langage »? Cette question passe, on le sait - et je ne pourrais trop épiloguer sur ce sujet - par toute une crise de la représentation, d'une reconfiguration de l'auteur et du lecteur, bref de la ruine d'un certain rapport à la langue et à la tradition (à la langue comme porteuse de tradition) ${ }^{19}$.

16. Alain Bergala, Voyage en Italie, p. 55.

17. Alain Bergala, Voyage en Italie, p. 49-50.

18. Cité dans Hanna Arendt, Between Past and Future, p. 7.

19. «Ruines vrai refuge enfin vers lequel d'aussi loin par tant de faux.» (Samuel Beckett, «Sans » [1969], dans Têtes-mortes, Paris, Éditions de Minuit, 1972 [1967], p. 69) 


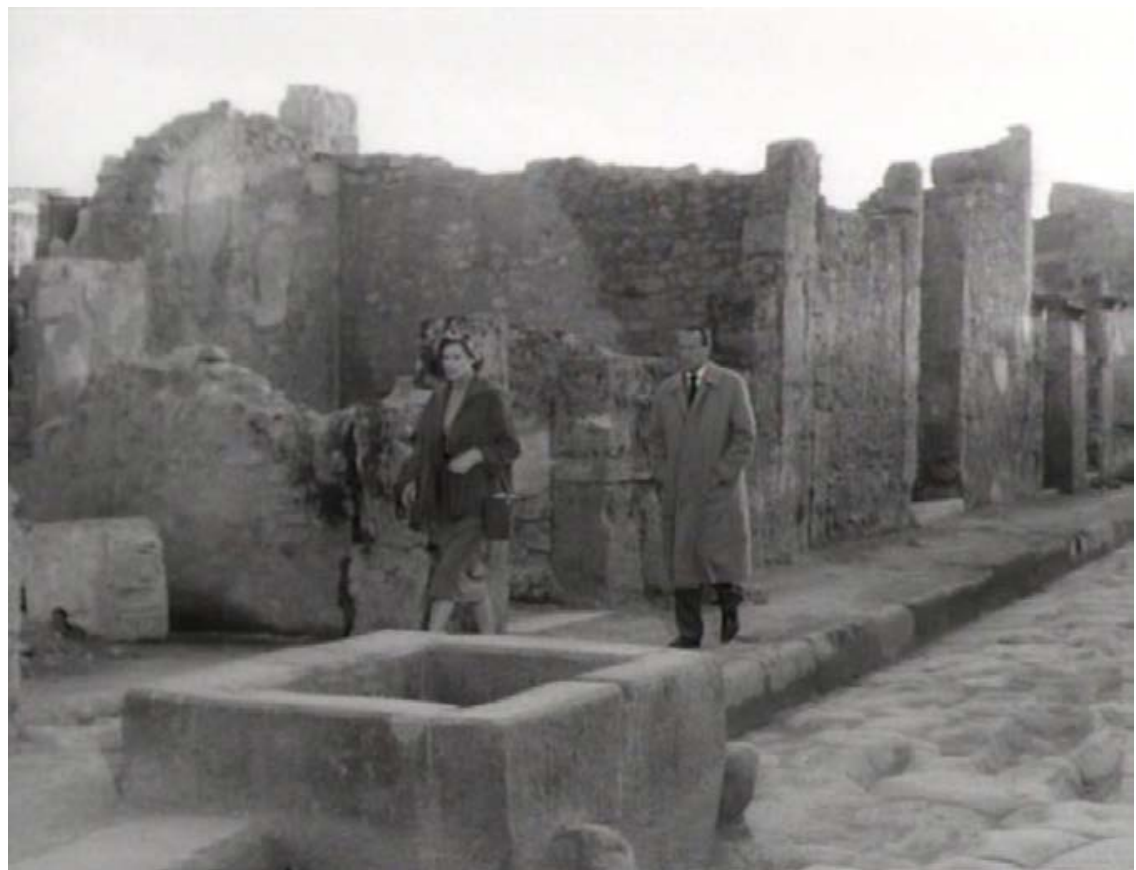

Fig. 7. Roberto Rossellini, Viaggio in Italia, 1954 (avec l'aimable autorisation de Renzo Rossellini).

Ce que Rossellini refait pour son compte, c'est le tracé de cette question: comment faire l'économie de la ruine, de la perte de sens, de la rupture ontologique entre soi, le langage et le monde, à partir d'une inquiétude et d'un ébranlement du langage et du récit.

Mais en réalisant Viaggio in Italia, ne pourrait-on pas dire que, d'une certaine façon, Rossellini reprend et reprise, en s'y inscrivant ne fût-ce qu'en porte-à-faux, une longue tradition littéraire des voyages en Italie qui, de Montaigne à Chateaubriand, de Jensen à Freud, de Goethe à Stendhal, de Ruskin à Proust, s'est toujours articulée sur la réinscription d'une disparition, bref d'une reprise conçue à partir d'une rupture (oubli, mise en ruine, expression d'un écart éventuellement reconductible avec le passé).

De ce point de vue, on serait parfois surpris de reconnaître dans Viaggio in Italia, avec des déplacements stratégiques, un certain nombre de topiques courantes à cette littérature du voyage. Il ne faut pas être un trop fin onomaste pour repérer dans le choix des noms des personnages, Joyce, Homère, une volonté, fût-elle flottante, de faire circuler un certain intertexte littéraire, quand on sait 
que James Joyce a séjourné pendant plusieurs années à Rome et à Trieste, et que le voyage de l'oncle Homère, tel qu'il est raconté dans le film, est une très lointaine évocation de L'Odyssée.

Le littéraire est déjà présent dans les vers que Katherine rapporte, ceux d'un jeune poète qu'elle avait connu avant son mariage ${ }^{20}$ :

Temple of the spirit, no longer bodies but pure ascetic images

Compared to which mere thought seems flesh, heavy, dim.

Ces vers resurgissent dans la mémoire de Katherine sur la terrasse à Naples et, une seconde fois (en voix off) dans la grotte des Sibylles. On sait que les voyages en Italie, et le spectacle des ruines en particulier, ont toujours été l'occasion d'une mise en mouvement de la mémoire et de l'imagination, et qu'ils ont souvent servi, chez Freud entre autres, de métaphore pour représenter les lieux de mémoire et la survivance des traces mnésiques ${ }^{21}$. Or, c'est bien parce que la mémoire, comme les paysages, ont été empreints, qu'ils reviennent sous forme de traces du passé, de temps retrouvé, des profondeurs archéologiques du passé22.

À cela nous pourrions ajouter que les voyages en Italie sont aussi des mises en texte qui retextualisent un espace, souvent déjà textualisé par la tradition littéraire: Du Bellay, dans ses Antiquités de Rome, reprenant des motifs de Pétrarque; Chateaubriand rethématisant le voyage de Goethe; Jensen dans Gradiva reprenant à Gautier certains éléments de Arria Marcella, sa fantaisie archéologique à Pompéi, qui seront ensuite redéployés par la lecture qu’en fera

20. D'ailleurs, Alain Bergala rappelle que l'anecdote que rapporte Katherine sur la terrasse au sujet de ce jeune poète tuberculeux, est une reprise évidente d'un passage de la nouvelle The Dead de James Joyce. Voir Alain Bergala, Voyage en Italie, p. 66, n. 27.

21. Sigmund Freud, Délire et rêves dans la Gradiva de Jensen, trad. Marie Bonaparte, Paris, Éditions Gallimard, coll. «Idées», 1971 [1907] et tout particulièrement Sigmund Freud, Malaise dans la civilisation, trad. Charles et Jeanne Odier, Paris, Presses universitaires de France, coll. «Bibliothèque de psychanalyse », 1992 [1929], p. 12-15. Voir également sur ce thème chez Freud, Philippe Dubois, "Palimpsestes», dans Anamnèse, Montréal, Dazibao, 1989, p. 17-27, et Jacques Derrida, Mal d'archives. Une impression freudienne, Paris, Éditions Galilée, coll. «Incises», 1995.

22. On se rappellera certains passages du Temps retrouvé de Proust, ou encore du texte de Walter Benjamin, dans ses Chroniques de Berlin où il reprend explicitement une métaphore archéologique : «He who seeks to approach his own buried past must conduct himself like a man digging. This confers the tone and bearing of genuine reminiscences.» (Walter Benjamin, «A Berlin Chronicle» [1932, 1970], dans Reflections, p. 26) 
Freud; Proust liant à une Venise totalement ruskinisée l'expérience de la mémoire involontaire et du temps retrouvé, etc. En ce sens, Rossellini, dans la mesure où il emploie à son tour des stratégies de réappropriation et de mise en scène d'un paysage toujours déjà ouvré par la tradition, réécrit et retrace à sa manière un espace discursif thématisé par la littérature.

Dans ces voyages en Italie, les ruines apparaissent comme un sujet de fascination pour les écrivains et les peintres et ont suscité, au courant des siècles, des postures très diverses qui recoupent et informent, transversalement, toute l'histoire de la littérature, et qui ne sont pas indifférentes au film de Rossellini. Les ruines, en effet, reflètent le présent en l'abîmant ou en le déroutant et appellent un retour sur soi. Elles libèrent la mémoire ou révèlent la fissure du moi. Elles se donnent également (depuis le XVIII ${ }^{\mathrm{e}}$ siècle, chez Diderot et, en particulier, chez les romantiques), comme paysage affectif qui affecte le sujet et qui se trouve, en retour, affecté dans sa description par le sujet. Enfin, depuis le $\mathrm{XIX}^{\mathrm{e}}$ siècle au moins, elles se présentent comme une image réflexive de la création artistique ${ }^{23}$.

Dans la tradition littéraire, on pourrait dire que le voyage en Italie, eu égard aux ruines, a consisté, entre autres choses, en un travail de réinscription mémoriale sensible, historique, d'une présence absente, d'un Esprit (du temps, d'une époque, d'un Âge, dirait Ruskin), libéré de sa forme, ou encore d'une forme libre qui délie l'esprit et permet les méditations. La forme lacunaire, défonctionnalisée de la ruine représente un Esprit ayant résisté au temps, ou montrerait le destin de toutes choses soumises au temps. Les ruines inspireraient alors, parce qu'en elles souffle un âge de l'unité du monde (romain en l'occurrence), ou encore, parce que la «mise en ruine » libère un ensemble de traits proprement esthétiques (ombres, non finito, libre jeu des formes, etc.), que le bâtiment conservé ne possède pas.

Mais qu'en est-il de la temporalité des ruines? Dans la mesure où elles trouveraient leur précondition dans une défonctionnalisation, ou une dysfonction de leur fonction d'origine (lieu de culte, bains publics, théâtre, etc.), les ruines nous lèguent un passé qui à la fois continue et dépasse notre présent. La

23. Sur toutes ces questions et sur la (re)textualisation des paysages, voir l'ouvrage méticuleux de Marie-Madeleine Martinet, Le voyage d'Italie dans les littératures européennes, Paris, Presses universitaires de France, 1996, ainsi que l'ouvrage de Roland Mortier, La poétique des ruines en France. Ses origines, ses variations, de la Renaissance à Victor Hugo, Genève, Librairie Droz, 1974. 
médiation que les ruines offriraient aurait ceci de paradoxal qu'elle re-présente un héritage (on parle volontiers des ruines comme faisant partie du patrimoine mondial) qui ne peut être reçu et saisi que par l'écart radical dans lequel elles s'exposent pour notre temps. Elles ne font partie de notre héritage culturel, bien souvent, qu'en devenant autre chose que ce à quoi elles étaient destinées, dans un combat entre nature et histoire, entre matière et esprit, entre lieu (action) et paysage (arrêt de l'action), entre immeuble et œuvre d'art. Elles rendent visibles une épaisseur du temps, une stratification de passés coexistants, contenant à la fois le travail et la perception des hommes qui ne sont plus et le labeur, toujours en œuvre, de la nature.

Simmel, dans son essai Les ruines, a bien analysé la façon par laquelle un ensemble architectural, en tombant en ruines, déconstruit l'œuvre humaine et entre en rapport dialectique avec le travail de la nature : «C'est tout l'attrait des ruines de permettre qu'une œuvre humaine soit presque perçue comme un produit de la nature ${ }^{24}$.» Les ruines, telles que nous les percevons, telles qu'elles apparaissent aux personnages de Rossellini, seraient alors la manifestation d'un écart, la révélation d'un «temps pur» auquel on participe, mais qui nous déborde infiniment, et auquel on ne se raccroche qu'à se situer dans cette béance du temps. Marc Augé, en réfléchissant sur les rapports entre ruine et histoire, avançait l'idée selon laquelle

[...] la nature, en ce sens, abolit non seulement l'histoire mais le temps. Les ruines ajoutent à la nature quelque chose qui n'est déjà plus de l'histoire mais qui reste temporel. [...] Le paysage des ruines, qui ne reproduit intégralement aucun passé et fait intellectuellement allusion à de multiples passés, doublement métonymique en quelque sorte, propose au regard et à la conscience la double évidence d'une fonctionnalité perdue et d'une actualité massive, mais gratuite. [...] Le «temps pur ", c'est ce temps sans histoire, dont seul l'individu peut prendre conscience et dont le spectacle des ruines peut lui donner fugitivement l'intuition ${ }^{25}$.

Aussi, au-delà d'un certain intertexte littéraire, il faut bien voir que Rossellini n'entend pas refaire le Voyage en Italie de Chateaubriand ou de Taine (cela n’est plus possible). Mais il peut redéployer une expérience du temps, de la

24. Georg Simmel, «Les ruines. Essai d'esthétique » [1907], dans La parure et autres essais, trad. Florence Vinas, Paris, Éditions de la maison des sciences de l'homme, 1998, p. 113.

25. Marc Augé, «Le temps et l'histoire», dans Le temps en ruines, Paris, Éditions Galilée, coll. «Lignes fictives», 2003, p. 39-40. 
survivance du temps, de sa résurgence fulgurante, de son «intuition fugitive ». Ce qui se trouve révélé, ce qui resurgit en tant que «temps pur», c'est alors, comme l'écrit Bergala, «une appartenance à l'Espèce, qui n'est pas recyclable dans une petite ou dans la grande Histoire». La survivance, telle qu'il faut l'entendre ici, est une expérience du temps qui refait surface sous forme de traces qui «regardent les vivants de façon énigmatique ${ }^{26}$ ».

C'est sans doute une telle survivance comme expérience d'un temps, à la fois composite et instantané, qu'expose Rossellini, et qui rejoint la définition de l'image que donne Georges Didi-Huberman, comme «cendre vivante », comme «fossile vivant ${ }^{27}$ ». Devant le couple désenseveli de Pompéi, le couple est renvoyé à lui-même, mais, plus essentiellement, est sommé de reconnaître sa coupure, la brèche dans laquelle il se meut, qui est signe, en même temps, d'une appartenance paradoxale au monde et au temps.

Le sens du reste devient alors le sens d'une perte de sens du monde, et forcerait à reconnaître, hors de tout ton chagrin et nostalgique, que toute perte est une chance de sens, puisqu'elle révèle dans sa complexité ce qui a été perdu. Si l'on peut parler de transmission et d'héritage ici, ce n'est qu'en relisant cette perte de sens comme ce dont ils héritent, et qui sera à redécouvrir continûment.

Voilà cinquante ans, Rossellini imprimait les premiers mètres de film d'une de ses plus grandes ouvres. Sans doute, en voyant ce film aujourd'hui, nous sommes à même de constater une autre modalité de la transmission, plus subtile, que ce film nous permet de penser: celle de sa propre trace, de sa propre empreinte de lumière, fixée sur une pellicule, qui nous revient du temps. À l'instantanéité indicielle du couple figé dans la lave pompéienne, Rossellini savait qu'il ne faisait pas autre chose, en figeant, en momifiant (selon la métaphore bazinienne) le regard d'Ingrid Bergman et de George Sanders. Depuis, dépouillés de leurs oripeaux, de leur laque fictionnelle, il regardent, par-devant

26. Alain Bergala, Voyage en Italie, p. 35 .

27. Voir à ce propos l'étude de Georges Didi-Huberman sur Aby Warburg: Georges Didi-Huberman, L'image-survivante. Histoire de l'art et temps des fantômes selon Aby Warburg, Paris, Éditions de Minuit, coll. «Critique», 2002. 
nous, ce monde avec lequel ils ont cessé de faire lien. N'est-ce pas eux qui, maintenant, s'adressent à nous de façon énigmatique ${ }^{28}$ ?

Si le fait moderne est, suivant Deleuze, le «fait que nous ne croyons plus en ce monde» et que le lien entre l'homme et le monde s'est rompu, n'est-ce pas ce lien, en tant qu'il est rompu, qu'il faut restaurer comme objet de croyance?

Seule la croyance au monde peut relier l'homme à ce qu'il voit et entend. Il faut que le cinéma filme, non pas le monde, mais la croyance à ce monde, notre seul lien $[\ldots]$. Nous redonner croyance au monde, tel est le pouvoir du cinéma moderne (quand il cesse d'être mauvais) ${ }^{29}$.

N'est-ce pas ce que filme Rossellini, dans les rues de Naples, précisément la rencontre inattendue avec une croyance «en ce monde-ci », tel qu'il est? Audelà, et pour nous, c'est bien parce que le cinéma, tel que le pratique Rossellini, nous permet d'entretenir un lien de croyance avec ce monde, encore que délié et délité, qu'il nous permet de relire en lui ces espaces de frayage, ces marques de survivance et la contemporanéité de son propre passé qui résiste au temps, par le seul fait qu'il nous reste, du temps. C'est l'évidence que nous transmet ce film.

28. C'est l'idée que dévelope Laura Mulvey dans un article où elle constate: «The film itself is the final layer in the process which extends the materiality of the index into its relation with time, the preservation of the past and the image of the living after the dead. » (Laura Mulvey, «Vesuvian Topographies. The Eruption of the Past in Voyage to Italy», dans David Forgacs, Sarah Lutton, Geoffrey Nowell-Smith (dir.), Roberto Rossellini: Magician of the Real, Londres, British Film Institute Publishing, 2000, p. 108)

29. Gilles Deleuze, Cinéma 2. L'image-temps, p. 223. 